ALICE/PUB-98-28

December 15, 1999

\title{
A pattern recognition method for the RICH based HMPID detector in ALICE
}

\author{
D. Elia ${ }^{a,}$, N. Colonna ${ }^{a}$, D. Cozza $^{a}$, D. Di Bari ${ }^{a}$, A. Di Mauro ${ }^{b}$,

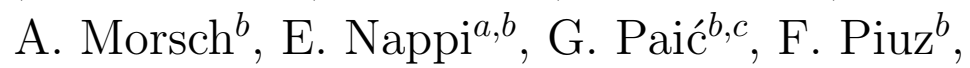 \\ S. Stucchi ${ }^{a}$ and G. Tomasicchio ${ }^{a}$
}

a. Dipart. I.A. di Fisica and INFN, Bari (Italy)

b. CERN, Geneva (Switzerland)

c. Ohio State University, Columbus, Ohio (USA)

(For the ALICE HMPID group and Collaboration)

\begin{abstract}
A pattern recognition method developed for the High Momentum Particle IDentification (HMPID) detector in the ALICE experiment at CERN is presented. The algorithm is based on the Hough transform with a mapping of the pad coordinate space directly to the Cherenkov angle parameter space.

Cherenkov angle reconstruction has been studied as a function of different particle densities in the photodetector using real data taken in the ALICE tests at the CERN SPS: a satisfactory resolution can be achieved even in events where the occupancy reaches more than $12 \%$, which is the situation we may be confronted with in central $\mathrm{Pb}-\mathrm{Pb}$ interactions at LHC.
\end{abstract}

\section{Introduction}

The HMPID detector in ALICE is devoted to the detection of charged particles $(\pi, \mathrm{K}$ and $p)$ in the momentum range between 1 and $5 \mathrm{GeV} / c[1]$. It consists of a single arm RICH based Cherenkov detector, with seven modules $\left(1.3 \times 1.3 \mathrm{~m}^{2}\right.$ each $)$ arranged in a barrel section about $5 \mathrm{~m}$ far from the beam

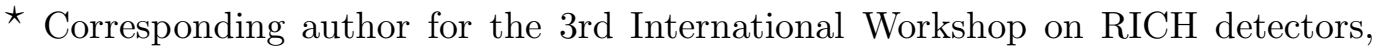
RICH '98, Ein Gedi (Israel), Dead Sea - November 15-20, 1998

Preprint submitted to Elsevier Preprint

16 March 1999 
line: details about detector design and technical features can be found in $[2,3]$. Charged track impacts and incidence angles at the RICH photocathode will be provided by the ALICE TPC with satisfactory resolution[4]. The highest expected charged particle multiplicity in central $\mathrm{Pb}-\mathrm{Pb}$ collisions in ALICE (about 8000 particles per unit of rapidity) corresponds to a maximum occupancy of about $12 \%$ [5]; consequently the recognition of Cherenkov patterns (which also depends on the track incidence angle) and the Cherenkov angle reconstruction itself figure out as very complex tasks.

The presented Cherenkov angle resolution has been obtained using an "Houghlike" pattern recognition method applied to samples of various particle densities and incidence angles.

\section{Data samples}

Data samples of different track densities have been obtained by randomly superimposing a fixed number of single track (beam) events on a pad map of the dimensions of the ALICE prototype module: these events were taken from data collected in the ALICE test at the CERN SPS in the last two years with a $340 \mathrm{GeV} / c$ pion beam[6]. Inclined tracks and different detector settings for the RICH-MWPC high voltage (gain) and proximity gap size (ring radius) have also been taken into account, as resumed in table 1.

\begin{tabular}{|c|c|c|c|c|}
\hline \hline Sample & ring radius & density $\left(\mathrm{p} . c l e s / \mathrm{m}^{2}\right)$ & $\mathrm{HV}$ & inc. angle \\
\hline \hline 1 & $155 \mathrm{~mm}$ & $1 \rightarrow 50$ & $2100 \mathrm{~V}$ & $0^{\circ}$ \\
\hline 2 & $155 \mathrm{~mm}$ & $1 \rightarrow 50$ & $2050 \mathrm{~V}$ & $0^{\circ}$ \\
\hline 3 & $155 \mathrm{~mm}$ & $1 \rightarrow 50$ & $2000 \mathrm{~V}$ & $0^{\circ}$ \\
\hline 4 & $122 \mathrm{~mm}$ & $1 \rightarrow 50$ & $2100 \mathrm{~V}$ & $0^{\circ}$ \\
\hline 5 & $122 \mathrm{~mm}$ & $1 \rightarrow 50$ & $2050 \mathrm{~V}$ & $2.5^{\circ}, 5^{\circ}, 7.5^{\circ}$ \\
\hline \hline
\end{tabular}

Table 1: Samples of data from SPS test-beam, used for the analysis.

The highest analyzed density (50 p.cles $/ \mathrm{m}^{2}$ ) has been calculated[7] to correspond to an occupancy of $14 \%$, even slightly bigger than the maximum expected for ALICE.

\section{Reconstruction and analysis of Cherenkov signal}

The signal recorded by the pad readout of the MWPC consists of the coordinates of hit pads and the amount of charge induced on each of them. The 
general input for the pattern recognition in ALICE will be a global pattern where single Cherenkov rings can hardly be identified by eye, and a map of impacts of the tracks extrapolated from the TPC to the RICH cathode with their momentum and incident angles.

In order to extract from the described cluster distribution and track impact information the Cherenkov signal for a chosen track the following steps have been applied:

- to define a "fiducial zone" on the detector plane where the Cherenkov photon clusters emitted by the chosen track of known momentum and incidence angle are observed;

- to associate to each cluster in the fiducial area a "Cherenkov angle" of emission in the radiator as if it were produced by the track.

The latter task is performed using a geometrical backtracing algorithm described in [7], essentially based on the fact that from a cluster centroid (or from a hit pad location) we can reconstruct the Cherenkov angle under which the photon causing it could have been emitted if it had belonged to the chosen track.

As a first step in the analysis procedure single track events at the SPS have been processed in order to obtain the Cherenkov angle resolution in absence of background coming from other tracks. The photon angle distribution obtained in this way can be described with a gaussian, giving a per photon resolution $\sigma_{\eta_{c}} \approx 12.8 \mathrm{mrad}$. By averaging $\eta_{c}$ for all photons, the Cherenkov angle per track $\theta_{c}$ can be obtained, giving a per track resolution $\sigma_{\theta_{c}} \approx 3.9 \mathrm{mrad}$.

The analysis of single ring events has shown that the average number of photoelectrons per track is about 14 with a corresponding average cluster size (i.e. hit pads per photoelectron) of about 1.5[6]. Although at first sight it seems advantageous to use the cluster centroid method in the backtracing (for a better localization) it has been observed that this method, even at moderate densities, leads to a reduction and biasing of the Cherenkov signal because of the partial overlap of clusters from different tracks[7]. For this reason we have analyzed the multi-ring events taking into account simply the hit pads, assuming as photon-pad location the middle of the pad itself.

Starting from the centroid of the cluster belonging to the track impact (MIP) and knowing the detector parameters, a wide fiducial region is defined around the MIP itself, where all photons relevant to that track may be found: in this analysis all pad hits corresponding to photon angles between 0.550 and 0.750 rad have been backtraced discarding, of course, those belonging to clusters with too high charge (other MIPs). In fig. 1 the white distributions correspond to the photon $\eta_{c}$ spectra obtained for all analyzed MIPs in samples at different particle densities.

These distributions show two distinct components: a peak around the Cherenkov angle produced by photons belonging to the tracks analyzed, and a continuous background belonging to hits produced by photons associated to other tracks 
and falling in the fiducial zone analyzed.

To describe this background, the following analytical expression

$$
\frac{d N_{b k g}}{d \eta_{c}} \sim F_{b k g}\left(\eta_{c}\right)=\operatorname{tg} \theta\left(1+g^{2} \theta\right) \frac{n_{C_{6} F_{14}}}{n_{C H_{4}}} \frac{\cos \eta_{c}}{\cos \theta}
$$

can be worked out from geometrical considerations. In equation (1) the refraction indices $n_{C_{6} F_{14}}$ and $n_{C_{H_{4}}}$ have been assumed constant and $\theta$ is the polar angle of the photon at the photocathode given by

$$
n_{C H_{4}} \sin \theta=n_{C_{6} F_{14}} \sin \eta_{c}
$$

Equation (1) fits well the real background at all particle densities, as shown by the dashed curves in fig.1.

The expected rising contribution of the background at larger angles would bias in any pattern recognition the results towards higher angles. This has been taken into account weighting each photon in the $\eta_{c}$ spectrum by

$$
W_{b k g}\left(\eta_{c}\right)=1-\frac{R_{b k g}\left(\eta_{c}\right)}{N_{p h}\left(\eta_{c}\right)}
$$

where $R_{b k g}\left(\eta_{c}\right)$ is the expected amount of background at any $\eta_{c}$ calculated by using equation (1) and properly normalized as reported in [7]. The dark distributions in fig.1 have been obtained by entering each photon-pad with the corresponding weight calculated by equation (2).

The pattern recognition for the HMPID, on the track level, has been implemented using the Hough transform technique to extract the mean Cherenkov angle per track as a parameter to be estimated in a transformed parametric space. This is achieved by analysing the parameters which characterize these patterns (i.e. the Cherenkov angle of each photon for the analyzed track, in this case) and looking for local maxima in a feature parameter space: the main advantage of the Hough transform is that it is relatively unaffected by topological gaps in curves and by high noise background in spot-like images $[8,9]$. Our basic approach is to apply a Hough transform method which has been modified to accomodate the fact that the signal in the $\eta_{c}$ coordinate has an intrinsic width. To account for that width we apply a sampling band approach that consists in sliding a window of a width comparable to the width of the signal over the $\eta_{c}$ spectrum. At each step in the sliding, the number of weighted photon-pads found within the window is counted: the most populated correlation band is called Hough selected band[7]. 


\section{Results}

The output response of the Hough analysis procedure, per each MIP, consists of the photon-pads falling into the Hough selected band: we call those "Hough selected photon-pads" and their number will be indicated by $N_{p h}^{H o u g h}$. Furthermore the band selection performed by the Hough method takes into account the background: then for each Hough selected photon-pad a background related weight is also available.

Finally, the pattern recognition provides this global output:

$$
N_{p h}^{\text {Hough }} \quad\left(\eta_{c}(i), W_{b k g}\left(\eta_{c}(i)\right), \quad \mathrm{i}=1, N_{p h}^{\text {Hough }}\right)
$$

This information can be used to provide a better estimation for the MIP Cherenkov angle as:

$$
\left\langle\theta_{c}\right\rangle=\frac{1}{W_{\text {tot }}} \sum_{i=1}^{\substack{\text { Hough } \\ \text { Hh }}} W_{b k g}\left(\eta_{c}(i)\right) \eta_{c}(i) \quad W_{\text {tot }}=\sum_{i=1}^{N_{p h}^{\text {Hough }}} W_{b k g}\left(\eta_{c}(i)\right)
$$

and the calculation is iterated, discarding photon-pads with $\eta_{c}$ by more than a defined number of sigmas from the $\left\langle\theta_{c}\right\rangle$ value. Finally, by iteration the best estimate for the current MIP angle $\theta_{c}^{M I P}$ is obtained.

In fig.2(a) the $\theta_{c}^{M I P}$ distribution at 50 p.cles $/ \mathrm{m}^{2}$ is shown and fig.2(b) resumes (white triangles) the behaviour of the corresponding sigmas ( angle resolution" per MIP) versus the particle density itself.

The same analysis procedure has also been applied for event samples (2) and (3) in table 1, i.e. overlapped beam events collected at the SPS H4 beam in 1997 and 1998, lowering the RICH-HV from $2100 \mathrm{~V}$ to $2050 \mathrm{~V}$ and $2000 \mathrm{~V}$ : the results are summarized in fig.2(b). No significant difference is observed for the two higher HV values, while a degradation is observed at $2000 \mathrm{~V}$, probably due to some photon losses at that voltage.

The behaviour of $\sigma_{\theta_{c}}$ versus particle density is reported in fig.3(a), where the results for samples (1) and (4) are compared. The better resolution obtained for smaller $R$ reflects the fact that the overlap of different patterns is smaller for smaller rings, hence the underlying noise background contribution is smaller. The analytical relation between the Cherenkov angle resolution and the corresponding momentum limit for $3-\sigma \pi-K$ separation is plotted in fig.3(b): it can be noted that the performance of the method provides a track by track separation up to more than $2.5 \mathrm{GeV} / \mathrm{c}$ for the most violent events anticipated in ALICE. Assuming the mean multiplicity of the recorded events to be about half the maximum value, the present analysis sets the $3-\sigma \pi-K$ separation value to $\sim 3 \mathrm{GeV} / c$.

The performance of the method has also been tested for tracks hitting the photocathode with some angle with respect to the normal direction (samples 
(5) in table 1). In fig.4 examples of rings produced by those tracks are shown. This analysis has been performed rejecting $1 / 4$ of the ring fiducial area for $2.5^{\circ}$ and $5^{\circ}$ and $1 / 3$ for $7.5^{\circ}$, as illustrated in fig.4. Those cuts give the best angle resolutions at the moment by removing, from the analysis, regions with high background contamination in the Cherenkov signal.

Finally fig.5 shows the evolution of the angle resolution going from normal incidence to $7.5^{\circ}$, which is close to the maximum expected in ALICE for the present HMPID design[5].

\section{$5 \quad$ Summary and conclusions}

The results of the pattern recognition method presented show that the best conditions for the HMPID detector are reached at the highest HV, i.e. 2100 $\mathrm{V}$ and at the smallest radius of $122 \mathrm{~mm}$. At the maximum expected particle density, a Cherenkov angle resolution of $\sim 6 \mathrm{mrad}$ for normal track incidence angle can be obtained, corresponding to a $3 \sigma \pi$-K separation at $2.7 \mathrm{GeV} / c$. This resolution degrades when larger incidence angles are analyzed, requiring the introduction of other parameters and further tuning in the pattern recognition; future developments will focus on this fine tuning.

\section{References}

[1] ALICE Collaboration, Technical Proposal, CERN/LHCC 95-71.

[2] ALICE HMPID group and Collaboration, Technical Design Report, CERN/LHCC 98-19.

[3] F. Piuz et al., The HMPID system for the ALICE experiment at $L H C$, these Proceedings.

[4] D. Di Bari, D. Elia, E. Nappi and G. Paić, Internal Note ALICE/97-39.

[5] A. Morsch et al., Physics sources of noise in Ring Imaging Cherenkov Detectors, these Proceedings.

[6] A. Di Mauro et al., R\&D and test-beam studies of large area CsI-RICH prototype, these Proceedings.

[7] D. Cozza, D. Di Bari, D. Elia, E. Nappi, G. Paić and G. Tomasicchio, Internal Note ALICE/98-39.

[8] D.S. McKenzie, S.R. Protheroe, Patt. Recognition 23, No. 3/4, 283-290, (1990).

[9] E. Nappi, F. Posa and G. Tomasicchio, Computer Physics Comm., North Holland 66, 293-307, (1991). 


\section{Figure caption}

Fig. 1: Distributions of $\eta_{c}$ per photon-pad at different particle densities, with estimated background (dashed) and after background subtraction (dark) for sample (1).

Fig. 2: Final Cherenkov angle distributions per MIP at 50 p.cles $/ \mathrm{m}^{2}$ for sample (1) (a) and sigma evolution with density for samples (1), (2) and (3) (b).

Fig. 3: Cherenkov angle resolution versus density at different radii (a) and momentum limit vs. $\sigma_{\theta}$ relation (b).

Fig. 4: Examples of rings for inclined tracks.

Fig. 5: Angle resolution as a function of the track incidence. 

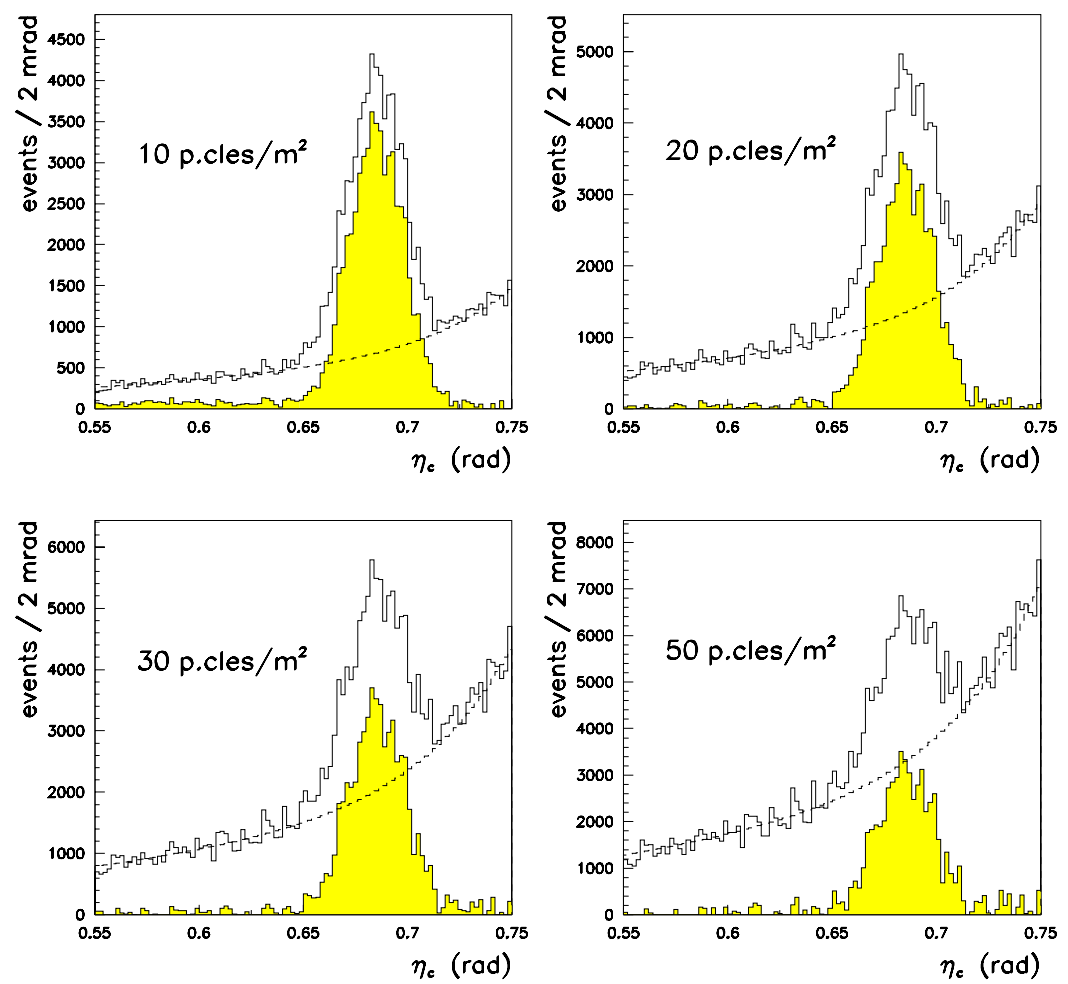

Fig. 1.
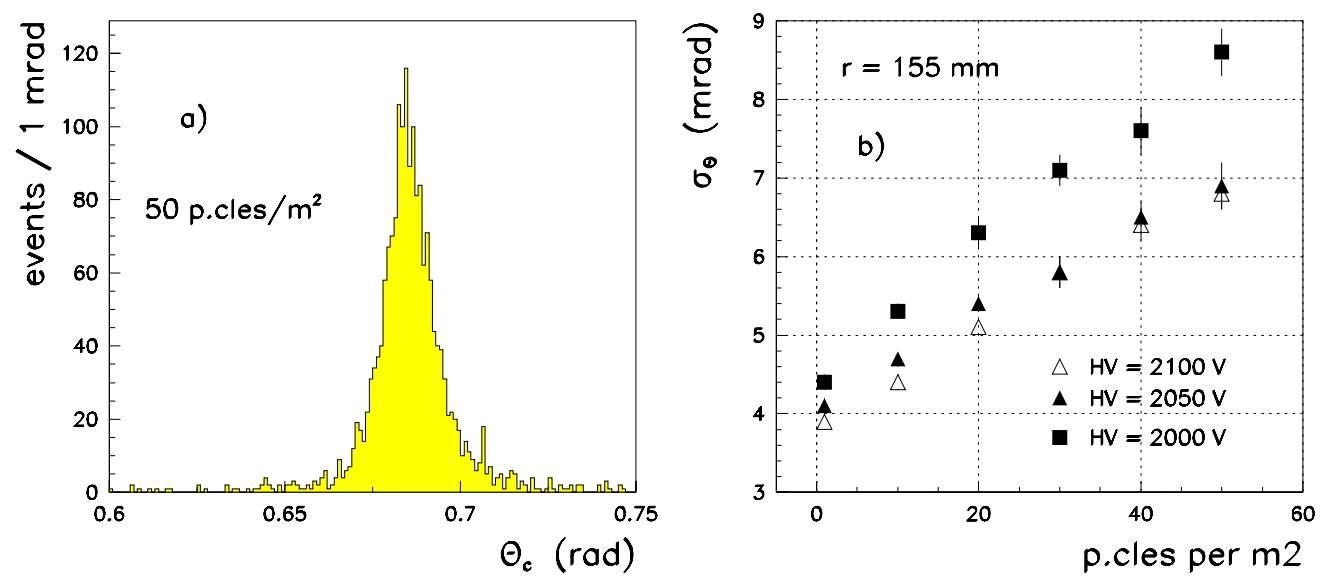

Fig. 2. 

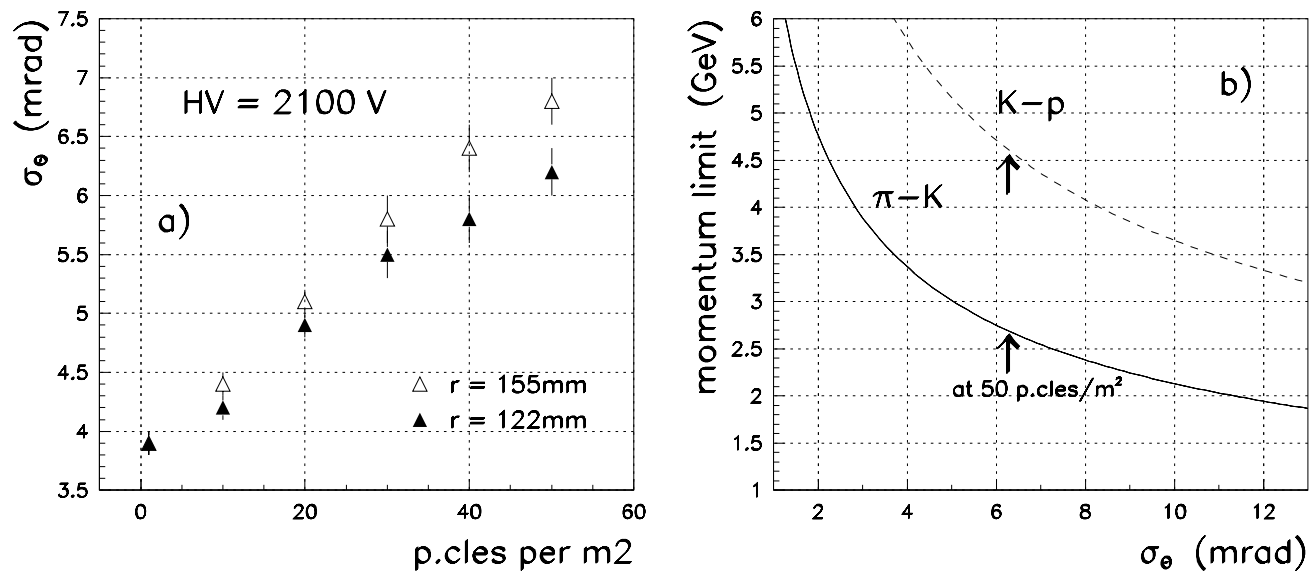

Fig. 3.
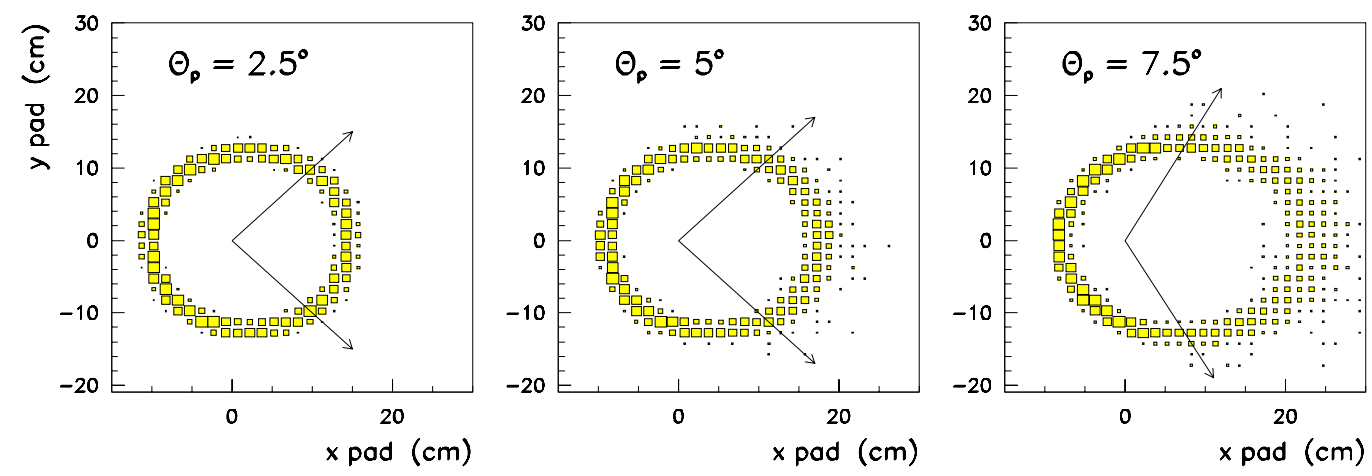

Fig. 4.

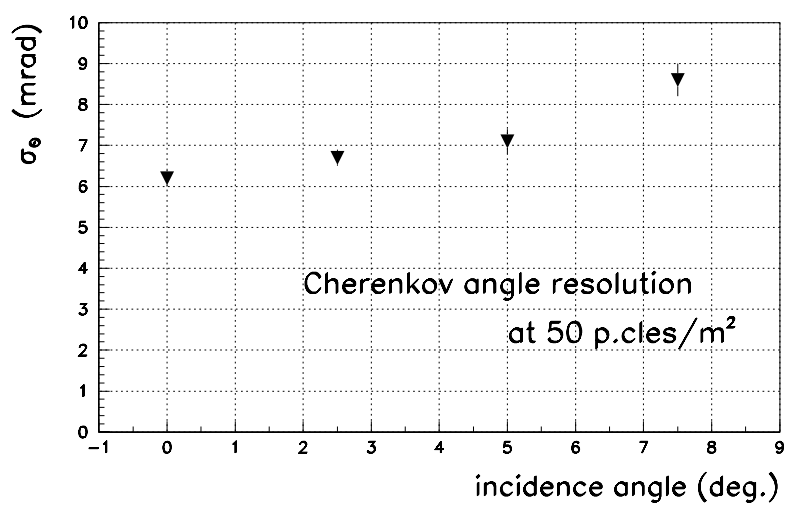

Fig. 5. 